\title{
A IMPORTÂNCIA DA FISIOTERAPIA NO PÓS-PARTO
}

\author{
Ingrid Silva Rodrigues ${ }^{1}$ \\ Tairo Vieira Ferreira ${ }^{2}$
}

RESUMO: Introdução: Durante o período de gestação e pós-parto o corpo das mulheres inúmeras transformações. Visto isso, uma das estratégias que pode ser utilizada é a fisioterapia pós-parto, portanto o presente trabalho apresenta a atuação do fisioterapeuta, algumas modificações e quais os benefícios do tratamento fisioterapêutico. Objetivo: Descrever os principais benefícios da Fisioterapia na saúde da mulher durante o período de pós-parto. Metodologia: Pesquisa de cunho bibliográfico, com critério de seleção onde foram selecionados so artigos através das palavras-chave, o critério de exclusão foi dado através da compatibilidade dos objetivos e com o tema requerido, totalizando 27 artigos utilizados para a revisão bibliográfica e 23 artigos descartados. Conclusão: A fisioterapia pós-parto é de suma importância para uma melhor recuperação, reeducar a função respiratória, estimular o sistema circulatório e prevenir tromboses, auxilia na redução da dor intra-abdominal, na diminuição de incontinência urinária, relaxamento ou tonificação muscular, melhoria do fluxo circulatório local, drenagem de líquidos, na cicatrização de diversos tecidos e no incentivo a regeneração dos mesmos.

Palavras- chave: Fisioterapia. Pós-parto. Gestação. ABSTRACT: Introduction: During the period of pregnancy and postpartum the woman's
body countless changes. In view of this, one of the strategies that can be used is postpartum physical therapy, so this work presents the role of the physical therapist, some modifications and the benefits of physical therapy treatment. Objective: To describe the main benefits of Physiotherapy on women's health during the postpartum period. Methodology: Search of a bibliographic nature, with selection criteria where 50 articles were selected through the keywords, the exclusion criterion was given through the compatibility of the objectives and with the required theme, totaling 27 articles used for the bibliographic review and 23 discarded articles. Conclusion: Postpartum physiotherapy is of paramount importance for a better recovery, re-educating respiratory function, stimulating the circulatory system and preventing thrombosis, helps to reduce intraabdominal pain, decrease urinary incontinence, relaxation or muscle toning, improvement of local circulatory flow, fluid drainage, healing of different tissues and encouraging their regeneration.

\footnotetext{
I' Formação acadêmica: Graduação. Instituição: Faculdade Unibras Rio Verde-GO. E-mail: ingridsilvaataides@gmail.com

²Faculdade Unibras Rio Verde-GO .E-mail: tairo@faculdadeobjetivo.com.br.
} 
Keywords: Physiotherapy. Postpartum. Pregnancy.

\section{INTRODUÇÃO}

Inúmeras alterações ocorrem no corpo da mulher, que tem como objetivo retornar os sistemas ao mais próximo possível ao pré-gravídico. O sistema urogenital, respiratório, cardiovascular, musculoesquelético, dentre outros, retornam aos poucos às suas funções anteriores. As mulheres necessitam de cuidados individuais e essenciais em relação à sua saúde, o que exige uma assistência multiprofissional de programas específicos para a saúde da mulher. (BELEZA; CARVALHO, 2009).

No pós-parto imediato domina a crise genital e prevalecem os fenômenos catabólicos e involutivos das estruturas hipertrofiadas ou hiperplasiadas durante a gravidez. Ocorrem as mais dramáticas alterações fisiológicas, assim como o surgimento de complicações. Enquanto o pós-parto tardio é o período em que todas as funções começam a ser influenciadas pela lactação. E no pós-parto remoto é um período com duração imprecisa, já que nas mulheres que não amamentam ele é breve (SOUZA, I999; REZENDE, 2002).

Necessita-se ressaltar que o período pós-parto é mais voltado para os cuidados ao recém-nascido, do que para a mãe que recentemente pariu. Em diversas situações as queixas realizadas por elas são consideradas irrelevantes devido ao processo de parturição. Portanto, pouco é encontrado na literatura científica em relação aos cuidados dispensados com a puérpera, especialmente quando mencionado a fisioterapia.

Este estudo abordará a atuação da fisioterapia nesse período. Como descrito são diversas as modificações que ocorrem no corpo da mulher. Existe a necessidade da realização de exercícios físicos supervisionados por profissionais da área, a fim de acelerar tal processo e auxiliar ao retorno das atividades diárias.

O objetivo geral deste estudo é compreender como o fisioterapeuta pode prevenir e tratar as alterações ocorridas no pós-parto e ressaltar a importância de aprofundar os conhecimentos e divulgação do tema.

Os objetivos específicos são:

- Discorrer sobre a necessidade da fisioterapia nesse período; 
- Conceituar o processo do pós-parto;

- Destacar os principais recursos fisioterapêuticos com evidência científica utilizados no pós-parto.

A estrutura do trabalho encontra-se organizada da seguinte maneira:

Título I, Alterações Fisiológicas na gestação;

Título 2, Avaliação fisioterapêutica;

Título 3, Atuação fisioterapêutica;

Título 4, Benefícios da fisioterapia pós parto;

Pretende-se, com esta pesquisa, ressaltar os principais acometimentos durante a gestação e descrever os benefícios do tratamento de fisioterapia em cada um deles.

\section{Revisão Bibliográfica}

\section{I.I Alterações fisiológicas na gestação}

A gestação é uma circunstância para a sobrevivência da vida humana, representando o período de formação de um novo ser. Esse período da vida da mulher inicia-se quando a fertilização se prolonga por um período por volta de 40 semanas, e termina com o parto. É uma fase em que acontecem alterações no estilo de vida, ocasionando mudanças na vida pessoal, conjugal e de toda a família. Consiste também em uma fase de preparação física e psicológica, para o nascimento e para a parentalidade. (COUTINHO et al., 2014). A mulher vive um período com alterações do aspecto biológico e psíquico e essas repercussões diferem de gestante para gestante e da idade gestacional (DA SILVA et al., 2015).

O volume sanguíneo no sistema cardiovascular aumenta gradativamente 35 a 50\% ao longo da gestação, e volta ao normal com aproximadamente 6 a 8 semanas após o parto. Ao ficar em pé há um aumento da pressão venosa nos membros inferiores decorrente do aumento do tamanho do útero e aumento na distensibilidade venosa. $O$ tamanho do coração se amplia e seu posicionamento fica mais elevado, devido ao movimento do diafragma. As alterações no ritmo cardíaco também são comuns no período gravídico. (BIM; PEREGO, 2007) 
As alterações na função pulmonar ocorrem devido aos problemas posicionais advindos do aumento uterino e estímulos hormonais de progesterona. A amplitude e a frequência das incursões respiratórias aumentam, o que promove uma elevação da capacidade inspiratória em virtude de uma diminuição no volume residual funcional. (VASCONCELOS et al., 2017).

As modificações hormonais na gravidez podem desenvolver alterações benignas da pele, sendo a hiperpigmentação. Esse escurecimento da pele pode acometer vulva e axilas, aréolas, parede abdominal e face. Quando a face é acometida denomina-se melasma ou cloasma gravídico, na parede abdominal tem-se um escurecimento da linha alba. Essa hiperpigmentação é resultante da ação dos hormônios melanotrófico, estrogênio e progesterona, elevados durante a gestação.

O sistema urinário tem-se um acréscimo do tamanho e do peso dos rins. $\mathrm{Na}$ gestante, há aumento do fluxo sanguíneo renal é em torno de $50 \%$ e da taxa de filtração glomerular entre 45 e 50\%. Existe também uma ascensão progressiva da bexiga pelo útero, que alonga o trígono vesical e retifica o ângulo uretrovesical, o que pode ser uma das justificativas para a ocorrência de incontinência urinária na gestação.

Diante as mudanças advindas da gestação destacam-se ainda as alterações no sistema musculoesquelético que são extremamente importantes para o fisioterapeuta. Os músculos abdominais são alongados até o ponto de seu limite elástico no final da gestação, já os músculos do assoalho pélvico precisam suportar o peso do útero, para isso desce até 2,5 cm até o final da gestação (BIM; PEREGO, 2007).

O constante crescimento uterino é considerado uma das principais causas da mudança na estática e dinâmica do esqueleto da gestante. Sua posição dentro da cavidade abdominal em conjunto com o aumento do peso e tamanho das mamas, são causas que contribuem diretamente para o deslocamento do centro de gravidade da mulher para cima e para frente, podendo dessa maneira acentuar a lordose lombar.

\section{I.2 Avaliação fisioterapêutica}

Durante o puerpério a atuação do fisioterapeuta pode ser iniciada logo após o parto, respeitando o período de repouso estabelecido e orientado pelo médico de acordo com o 
tipo de parto, geralmente varia entre seis e dose horas (SILVA, 20II). O atendimento do fisioterapeuta é iniciado através de coleta de dados, inspecionando o histórico da gestação e parto, são observados os dados vitais, resultados de exames físicos, o padrão de respiração, a mobilidade diafragmática, a simetria das mamas, a região inguinal, os maléolos, possíveis formações de trombos e presença de edemas e varizes e por fim presenças de dores e não dores (POLDEN E MANTLE, 2002).

Para realizar qualquer procedimento fisioterapêutico, é necessário averiguar o horário de procedimento do parto, respeitando as oito primeiras horas de repouso em casos de cesariana, e respeitar o estresse físico e emocional provocado pelo parto. $\mathrm{O}$ atendimento deve ser iniciado com a coleta dos dados pessoais e relacionados ao parto no prontuário. (LOTTI, 2017).

Antes de se realizar o exame físico, é importante conversar e analisar os possíveis desconfortos e dores. Durante esse contato é primordial avaliar também a postura e posicionamento no leito, para orientá-la na presença de forma mais eficiente. Além disso, o estado emocional e a receptividade às orientações também são observados. Após o fisioterapeuta deve aferir a pressão arterial (PA), frequência respiratória (FR) e frequência cardíaca (FC), pois a ocorrência de anormalidades pode limitar a conduta. (BARACHO, 2018).

A musculatura do assoalho pélvico (MAP) também precisa ser avaliada, independentemente do tipo de parto. Para isso, podem-se solicitar contrações da musculatura do assoalho pélvico e observar se o movimento que está sendo feito de forma correta. As mamas, por sua vez, devem ser inspecionadas quanto a sua aparência geral, observando características como simetria, condições mamilares e presença ou não de dores.

\subsection{Atuação fisioterapêutica}

A fisioterapia é necessária para uma melhor recuperação e seu papel consiste na recuperação, prevenção e tratamento de alterações. A atuação fisioterapêutica durante o pós-parto pode ser iniciada após o parto, respeitando apenas o período de repouso de seis horas para o parto normal e doze horas para o parto cesárea.

$\mathrm{O}$ atendimento é iniciado com a coleta de dados, da história da gestação e parto. $\mathrm{Na}$ 
avaliação são observados os dados vitais e realizado o exame físico, deve-se verificar o padrão respiratório, a mobilidade diafragmática e a expansibilidade torácica. (SOUZA, 1999).

A intervenção fisioterapêutica tem como objetivos: proporcionar e orientar quanto ao posicionamento no leito, reeducação da função respiratória, estimulação do sistema circulatório, restabelecer a função intestinal, reeducação dos músculos abdominais, reeducação da musculatura de assoalho pélvico, promover analgesia no local da incisão perineal ou cesárea e orientações gerais em relação aos cuidados com as mamas e quanto às posturas assumidas durante o cuidado com o bebê.

O fisioterapeuta deve orientar a paciente quanto a uma postura correta no leito, como, por exemplo, o decúbito lateral para facilitar a eliminação dos flatus, incentivar a deambulação precoce e evitar posturas antiálgicas, aliviando as tensões musculares e promovendo analgesia, estimulando sempre uma postura correta (SOUZA, 1999).

\subsection{Benefícios da fisioterapia no pós-parto}

A atenção à mulher no pós-parto é de suma importância para a saúde materna e neonatal. Os recursos fisioterapêuticos utilizados nesse momento promovem o alívio dos desconfortos desencadeados nessa fase, reduzindo desse modo os custos hospitalares e ainda diminui o tempo de internação e a utilização de medicamentos pelas pacientes.

O papel da fisioterapia no pós-parto consiste no tratamento, recuperação e prevenção nos diversos sistemas a fim de minimizar os efeitos indesejáveis das alterações fisiológicas. A fisioterapia nesse período promove diversos benefícios, tais como:

\section{I.4.I Redução da diástase dos músculos retos abdominais}

As diástases dos músculos retos abdominais é uma condição que pode acontecer tanto no período puerperal quanto no último trimestre gestacional, quando apresenta um comprimento superior a $3 \mathrm{~cm}$ a diástase é considerada como patologia, interferindo na capacidade de movimentação do tronco e da capacidade de estabilização da musculatura (BEZERRA; NUNES; LEMOS, 2011; RETT et al., 2009; SOUZA, 2002;). 
Mesquita et al (1999) afirma que um dos benefícios que a fisioterapia pós parto trás é a redução da diástase dos músculos retos abdominais, para comprovação realizou-se um estudo com 50 puérperas recrutadas na Maternidade-Escola Hilda Brandão da Santa Casa de Belo Horizonte (MEHB-SCBH), no período de aproximadamente 6 meses no ano de 1998. Foram separados dois grupos, cada grupo composto por 25 pessoas, um grupo com tratamento e o outro não. No grupo sem o tratamento foi realizada uma avaliação da diástase de 6 e 8 horas após o parto, e no grupo de tratamento foi realizada a mesma avaliação de 6 e 18 horas pós parto. Utilizou-se um paquímetro na mensuração da diástase nas avaliações. $\mathrm{O}$ resultado obtido foi que no período de 18 horas pós-parto, as 25 mulheres sem o tratamento apresentou uma redução da diástase de 5,4\% e o grupo de mulheres que receberam o tratamento foi de $12,5 \%$, em relação à primeira medida (6 horas após o parto), ou seja, foi comprovado que a eficácia e beneficio é de mais de $6 \%$.

\subsubsection{Alterações musculoesqueléticas}

Baracho (2007) e Polden; Mantle (2005) corroboram citando alterações que ocorrem durante o pós-parto quando afirmam que ocorrem diversas modificações e adaptações durante o período gestacional e isso se da por causa do crescimento e desenvolvimento do feto. Em alguns casos essas modificações podem causar limitações e dores durante as atividades de vida diária. Isso depende de vários fatores, ou seja, acontecerá de forma individual, de acordo com a extensão das articulações, com a força muscular, modelos de posições, fadiga dentre outros.

Camarão (2004) cita que trabalhando o corpo através de tratamento fisioterapêutico, o corpo é visto como um todo, integrando corpo e mente, um método que pode ser utilizado é o pilates, que aumenta a flexibilidade, reestrutura o corpo, aumenta o condicionamento mental e físico, aumenta o equilíbrio, alivia dores musculares, melhora da capacidade respiratória, correção postural, previne lesões e aumento da coordenação.

Endacott (2007) apresenta mais benefícios da fisioterapia nas alterações musculoesqueléticas:

Dentre os principais benefícios ele destaca: - Melhorar a estabilidade da musculatura postural e a resistência dos músculos do assoalho pélvico; Proporcionar excelente força dos músculos abdominais; - Diminuir/aliviar 
as dores com frequência; - Melhorar a circulação abdominal; - Garantir benefícios impares a saúde do seu bebê em desenvolvimento; Desenvolver uma maior conscientização sobre seu corpo; - Promover uma boa recuperação do trabalho pós-parto (ENDACOTT, 2007).

O tratamento fisioterapêutico proporciona inúmeros benefícios e pode ser trabalhado de forma dinâmica ou isoladamente, em áreas especificas ou globais.

\subsubsection{Incontinência urinária}

Já Conceição (2002) complementa especificando sobre incontinência urinaria, salientando que através da fisioterapia pode-se conseguir fortalecimento dos músculos do assoalho pélvico através de contrações ativas e assim fazendo inibição reflexa da contração do detrusor e aumentando o suporte uretral.

De acordo com Modotte et al deve-se considerar o tônus vaginal, a perineômetria e o ped test para avaliação antes de iniciar o tratamento de fisioterapia com as mulheres, pois quanto mais caracterizada for a incontinência urinária, melhores resultados serão obtidos.

O tratamento e a prevenção de forma fisioterapêutica, são essenciais para a melhora na realização das atividades e qualidade de vida e consiste na pratica de exercícios de fortalecimento da musculatura do assoalho pélvico. (MELO; FERREIRA, 2014).

\subsubsection{Assoalho pélvico}

De acordo com Endacott (2007) para diminuir e evitar alguns problemas de intestino e bexiga é necessário o fortalecimento dos músculos do assoalho pélvico (na camada superior formado pelos músculos levantadores do ânus - que se divide em músculo pubovisceral, músculo puboccígeo e músculo ileococcígeo - e músculo coccígeo, na camada muscular média sendo composta pelo músculo longitudinal do ânus e por fim na camada muscular inferior composta pelos músculos: esfíncter anal externo, bulbo cavernosos, isquio cavernosos, transverso profundo e superficial do períneo).

A parte fundamental na manutenção da continência urinária e no suporte dos órgãos pélvicos é a fáscia endopélvica e a musculatura estriada do assoalho pélvico (RIBEIRO \& ROSSI, 2000).

Os músculos fazem um trabalho extra quando saem do parâmetro considerado normal de atividade do individuo, então esse trabalho a mais que requer dos músculos é 
devido a necessidade de superar a carga exigida. Essa exigência de carga faz com que haja um aumento de força através da contração muscular e do estimulo da síntese de proteínas musculares. A recuperação e a construção de novas proteínas acontecem após um período de descanso, aumentando o diâmetro e força das fibras musculares (BRINCK \& NERY, 2006)

O beneficio que a fisioterapia trás com treinando de força está diretamente ligado com a potência muscular, os exercícios recomendados geralmente estão em função da força desenvolvida pelo músculo e da velocidade em que se executam os movimentos (SIMÃO, 2001).

Todos os exercícios de fortalecimento promovem: hipertrofia devido ao aumento no tamanho das fibras musculares, que acontece por aumento na síntese protéica e diminuição da degradação de proteínas, fenômeno que leva de seis a oito semanas para ocorrer; maior eficiência de recrutamento de fibras musculares pelos mecanismos de neurofacilitação (ou aprendizado motor); aumento da capacidade oxidativa e volume das mitocôndrias; aumento da densidade óssea, do conteúdo mineral ósseo e de hidroxiprolina; alterações nas células do corno anterior da medula espinhal e aumento da resistência de tendões e ligamentos (MACHADO, 2005).

Através da prática de exercícios terapêuticos específicos para o assoalho pélvico adquiri-se força muscular, conforme o princípio de que as repetições de movimentos aumentam a força muscular e proporciona efeitos benéficos como: manutenção e restauração da força, melhora do desenvolvimento, aumento da resistência, do relaxamento, da flexibilidade, da coordenação, das habilidades e da mobilidade (HILBERATH, 2006; RUBINSTEIN, 200I).

\section{METODOLOGIA}

Foi realizada uma revisão bibliográfica, durante o segundo semestre de 202I, embasada em livros, documentos acadêmicos, como: monografias, dissertações, teses e artigos científicos. Os estudos selecionados foram por meio de buscas em plataformas eletrônicas, tais como: Google Acadêmico, Biblioteca Virtual em Saúde (BVS) e Scientific Electronic Library Online (SciElO), datados a partir de 1999 sobre o tema escolhido. As palavras chave utilizadas para a pesquisa foram: profissionais de fisioterapia, atuação do fisioterapeuta no pós parto, métodos fisioterapêutico pós-parto, gravidez, gestação, pósparto, fisioterapia na incontinência urinária, incontinência urinária, fisioterapia no pós- 
parto, cinesioterapia pós-parto, assoalho pélvico, alterações músculo esqueléticas na gestação, redução da diástase dos músculos retos abdominais.

Foram selecionados 50 artigos através das palavras-chave, o critério de exclusão foi dado através da compatibilidade dos objetivos e com o tema requerido, totalizando 27 artigos utilizados para a revisão bibliográfica e 23 artigos descartados.

\section{CONCLUSÃO}

A gestação é entendida como um processo fisiológico acompanhado por uma sequência de adaptações que ocorrem no corpo da mulher após a fertilização. Tais alterações são internas e externas, necessárias e então esperadas nesse período. Dentre as modificações estão alterações musculoesqueléticas, geniturinária, respiratórias, gastrointestinais, nervosas, cardiovasculares e tegumentares, sendo considerado um misto entre biológico e psicossocial que interfere na imagem corporal, relacionamento com o parceiro e convívio familiar. (LEANDRO; SILVA; SILVA, 2017).

Pouco se encontra pessoas com conhecimento sobre a atuação da fisioterapia no pós-parto e seus benefícios. Sabe-se que profissionais atuam nessa área há um determinado tempo, no entanto não se encontra detalhadamente sobre essa fundamental assistência. Visto que fisioterapia é de suma importância para uma melhor recuperação, reeducar a função respiratória, estimular o sistema circulatório e prevenir tromboses, restabelecer a função gastrointestinal, promover analgesia da região do períneo e da incisão da cesariana, retomar o condicionamento cardiovascular, reeducar a musculatura abdominal.

Portanto, além das ajudas mencionadas, ainda oferece orientações sobre posturas corretas ao amamentar e nos cuidados com o bebê.

Coelho et al (202I), afirma que a fisioterapia pós-parto auxilia na redução da dor intra-abdominal, na diminuição de incontinência urinária, relaxamento ou tonificação muscular, melhoria do fluxo circulatório local, drenagem de líquidos, na cicatrização de diversos tecidos e no incentivo a regeneração dos mesmos.

Diante de todos os benefícios citados está justificado o porquê da importância dessa pesquisa, afora que, por exemplo, existem cerca de 1716 fisioterapeutas que trabalham pelo SUS apenas no Distrito Federal e cerca de 6235 fisioterapeutas no estado de Goiás de 
acordo com os relatórios de 2021 do departamento de Demandas de Coordenação Geral De Informação Da Atenção Primária, dentre esses mais de 7.000 profissionais nem 1o \% trabalha com fisioterapia pós-parto, até mesmo pela falta de conhecimento dos usuários e profissionais sobre os benefícios da fisioterapia pós-parto.

\section{REFERÊNCIAS}

BARACHO, E. Fisioterapia aplicada à obstetrícia, uroginecologia e aspectos de mastologia. 4. ed. rev. e ampliada. Rio de Janeiro: Guanabara Koogan, 2007.

BARACHO, Elza. Fisioterapia aplicada à saúde da mulher. 6. ed. Rio de Janeiro: Guanabara Koogan, 2018.

BIM, Cintia Raquel; PEREGO, Alline Lilian; PIRES-JR, Hugo. Fisioterapia aplicada à ginecologia e obstetrícia. Iniciação Científica Cesumar, v. 4, n. I, p. 57-61, 2007.

BRINCK, NB. NERY, TP. O treinamento de potência muscular em membros inferiores e a possibilidade do aumento de saltos em bailarinos contemporâneos. Disponível em: http://www.jvianna.com.br/jefe/artvin2_02.pdf. Acesso em: o8 de Nov de 2021.

CAMARÃO, T. Pilates no Brasil: corpo e movimento. $2^{2}$ reimpressão. Rio de Janeiro: Elsevier, 2004.

Coelho, S. R. de A. ., Oliveira, A. A. ., Feyh , F. A., Bezerra, L. da S. C. ., \& Siqueira , S. G. . (202I). FISIOTERAPIA NA PÓS GESTAÇÃO. RECIMA2I - Revista Científica Multidisciplinar - ISSN 2675-6218, 2(7), e27513. https://doi.org/10.47820/recima21.v2i7.513 Conceição JCJ, Mitrano PGG. Deficiência esfincteriana intrínseca da uretra. Femina 2002;30(6):38I-84.8

COUTINHO, Emília de Carvalho et al. Gravidez e parto: O que muda no estilo de vida das mulheres que se tornam mães? Revista da Escola de Enfermagem da USP, v. 48, n. spe2, p. 17-24, 2014 . 
DA SILVA, Luzenilda Sabina. Análise das mudanças fisiológicas durante a gestação: desvendando mitos. Revista Faculdade Montes Belos (FMB), v. 8, n,I, p. 3-16, 2015. ENDACOTT, J. Pilates para grávidas: exercícios simples e seguros para antes e depois do parto. I. ed. Barueri, SP: Manole, 2007.

HILBERATH, E. C. Exercícios Perineais Supervisionados e cone vaginal no tratamento da Incontinência Urinária Feminina. Disponível em: http//www.biblioteca.unesp.br. Acesso em: I4maio.2006.

IMÃO, R. Avaliação da Medida Simples da Potência Muscular Máxima pelo Fitrodyne: fidedignidade e exemplo de utilização prática (dissertação de mestrado). Rev. Brasileira de Atividade Física e Saúde, Londrina - PR, vol. o6, no 03, 2001.

LEANDRO, Jayanny Pereira; SILVA, Sara Gabriella Ferreira; SILVA, Cinara Karina Bezerra. A assistência fisioterapêutica prestada as gestantes durante o prénatal: uma revisão de literatura. 2017. Disponível em http://repositorio.asces.edu.br/bitstream/123456789/1236/I/artigo\%2ofinal.pdf> Acesso em: 03 de abril de 2021.

MACEDO, Neusa Dias de. Iniciação à pesquisa bibliográfica: guia do estudante para a fundamentação do trabalho de pesquisa. zed. São Paulo: Edições Loyola, 1994. MACHADO, A. Análise da força muscular em mulheres praticantes de musculação na fase menstrual e pós-menstrual. Disponível em: http://www.ucb.br. Acesso em: 30 mar. 2005.

MARINHO, A. C. N. Atuação fisioterapêutica no aleitamento materno e na intercorrência mamária. Fisioterapia Brasil. São Carlos, v. 5, n. 4, p. 317-320, 2004. MELO, Eliane Cristina de Alvarenga; FERREIRA, Laísa Cavalcante. A intervenção Fisioterapêutica na prevenção da diástase do músculo reto abdominal em gestantes. Revista Brasileira de Saúde Funcional, Cachoeira- BA, v.I, n. I, p.I8-30, Jun. 2014 
MESQUITA, Luciana Aparecida et al. Fisioterapia para redução da diástase dos músculos retos abdominais no pós-parto. Revista Brasileira de Ginecologia e Obstetrícia, [S.L.], v. 2I, n. 5, p. 267-272, jun. 1999. FapUNIFESP (SciELO). http://dx.doi.org/10.159o/soroo72031999000500004

Modotte WP, Moreira ECH, Dias R, Pascotini C, Sartorão CIF, Braga MA. Incontinência urinária: tratamento conservador. GO Atual 1999;8:6-13

POLDEN, M.; MANTLE, J. Fisioterapia em Ginecologia e Obstetrícia. r.ed. São Paulo: Santos, 2002

POLDEN, M; MANTLE J. Fisioterapia em ginecologia e obstetrícia. São Paulo, Santos: 2005 .

REZENDE, J. Obstetrícia. 9. ed. Rio de Janeiro: Guanabara. 2002. 15I4p.

RIBEIRO, R. M.; ROSSI, P. Diagnóstico clínico e radiológico da incontinência urinária de esforço. In: MONTELLATO, N.; BARACAT, F.; ARAP, S. Uroginecologia. São Paulo: Roca, 2000.

Silva, M. L. B. A atuação da fisioterapia no parto e pós-parto. 20II. Disponível em:https://portalbiocursos.com.br/ohs/data/docs/35/I2_A_atuaYYo_da_fisioterapia_no_ parto_e_pYs-parto.pdf Acesso em: 30 mar. 2011.

SOUZA, E. L. B. L. Fisioterapia Aplicada à Obstetrícia e Aspectos de Neonatologia. 2.ed. Belo Horizonte: Health. 1999. 354p. 